\title{
NUMERICAL COUPLING OF 2.5D RESERVOIR AND 1.5D WELLBORE MODELS IN ORDER TO INTERPRET THERMOMETRICS*
}

\author{
M. AMARA $\ddagger$ D. CAPATINA $\ddagger$ L. LIZAIK ${ }^{\S}$
}

\begin{abstract}
The paper deals with the numerical coupling of an axisymmetric reservoir model, governed by Darcy-Forchheimer's equation together with a nonstandard energy balance, and a quasi 1D wellbore model described by the compressible NavierStokes equations.
\end{abstract}

Keywords. Petroleum wellbore and reservoir; Heat transfer; Coupling DarcyForchheimer and Navier-Stokes; Mixed finite elements.

Introduction. Thanks to new technologies such as optical fiber sensors, the temperature in petroleum wellbores is now measured continuously in time and all along the well. Using these recordings, as well as a flowrate history at the bottom of the well, the hope is twofold : to predict the flow repartition between each producing layer and to estimate the virgin reservoir temperature. Note that a sharp estimate of the latter is crucial to better characterize the thermodynamic properties of the fluid in place.

In order to solve these inverse problems, a first step is to solve a forward problem modeling the flow of a monophasic compressible fluid in the reservoir and the wellbore, including thermal effects. Most of the existing reservoir simulators are either isothermal or neglect certain physical phenomena (such as Joule-Thomson's effect), which play an important role when small variations of temperature are to be interpreted. Other codes exist dedicated to the wellbore but they don't take into account the evolution of the temperature in the reservoir.

An axisymmetric reservoir model, notably including viscous dissipation and compressibility effect, and a quasi $1 \mathrm{D}$ vertical wellbore model were previously introduced and analysed in [1], respectively [2]. They were separately validated from theoretical and numerical points of view. In this paper, we present their coupling and validate the obtained code. The extension of this work to a multiphase flow is in progress.

1. The 2.5D reservoir model. The considered domain is a petroleum reservoir treated as a porous medium divided into several geological layers. Each layer is saturated with both a mobile single phase fluid and a residual formation water and is characterized by its own physical properties.

The governing equations are the mass conservation law and the Darcy-Forchheimer equation (Cf. [6]), to which we add an exhaustive energy balance, including the temperature effects due to the decompression of the fluid (Cf. [8]) and the viscous dissipation

\footnotetext{
*THIS WORK IS SUPPORTED BY TOTAL

†UNIVERSITY OF PAU, LMA, CNRS UMR 5142, AV. DE L'UNIVERSITÉ, 64013 PAU

¥UNIVERSITY OF PAU, LMA, CNRS UMR 5142, AV. DE L'UNIVERSITÉ, 64013 PAU

§TOTAL, CST JEAN FEGER, AV. LARRIBAU, 64018 PAU
} 
(Cf. [4]) and the Peng-Robinson state equation (Cf. [7]) :

$$
\left\{\begin{array}{l}
\phi \frac{\partial \rho}{\partial t}+\operatorname{div} \mathbf{G}=0 \\
\rho^{-1}\left(\mu \underline{\mathbf{K}}^{-1} \mathbf{G}+F|\mathbf{G}| \mathbf{G}\right)+\nabla p=-\rho \mathbf{g} \\
(\rho c)_{*} \frac{\partial T}{\partial t}+\rho^{-1}(\rho c)_{f} \mathbf{G} \cdot \nabla T-\operatorname{div} \mathbf{q}-\phi \beta T \frac{\partial p}{\partial t}-\rho^{-1}(\beta T-1) \mathbf{G} \cdot \nabla p=0 \\
\rho=\rho(p, T) .
\end{array}\right.
$$

Here above, $\phi$ is the porosity, $\mu$ the viscosity, $\lambda$ the thermal conductivity, $\beta$ the expansion coefficient, $F$ the Forchheimer coefficient (allowing us to take into account the kinematic energy losses) and $\underline{\mathbf{K}}$ the permeability tensor. $(\rho c)_{*}$ characterizes the heat capacity of a virtual medium, equivalent to the fluid and the porous matrix, while $(\rho c)_{f}$ symbolizes the fluid properties.

The unknowns are the specific flux $\mathbf{G}=r \rho \mathbf{u}$ (with $\mathbf{u}$ the Darcy velocity), the heat flux $\mathbf{q}=r \lambda \nabla T$, the pressure $p$, the temperature $T$ and the density $\rho$. We close the system by adding initial conditions for $p$ and $T$ and boundary conditions which apply either to $\mathbf{G} \cdot \mathbf{n}$ or to its dual variable $p$, respectively to $\mathbf{q} \cdot \mathbf{n}$ or $T$. Due to the geometry of the domain (a cylindrical vertical wellbore surrounded by the reservoir), the problem is written in axisymmetric form and next time-discretized by means of Euler's implicit scheme, leading at each time step $t^{n}$ to the following $2 \mathrm{D}$ linearized system :

$$
\left\{\begin{array}{l}
\frac{1}{r} \underline{\mathbf{M}} \mathbf{G}+\nabla p=-\rho^{n-1} \mathbf{g} \\
\frac{1}{r \lambda} \mathbf{q}-\nabla T=0 \\
r \frac{a}{\Delta t} p-r \frac{b}{\Delta t} T+\operatorname{div} \mathbf{G}=r \frac{a}{\Delta t} p^{n-1}-r \frac{b}{\Delta t} T^{n-1} \\
r \frac{d}{\Delta t} T-r \frac{f}{\Delta t} p+e \mathbf{G}^{n-1} \cdot \nabla T+l \mathbf{G}^{n-1} \cdot \nabla p-\operatorname{div} \mathbf{q}=r \frac{d}{\Delta t} T^{n-1}-r \frac{f}{\Delta t} p^{n-1} .
\end{array}\right.
$$

The density is updated by means of a thermodynamic module. The thermodynamic coefficients $a, b, d, e, f, l$ are computed at $t^{n-1}$ and the positive definite tensor $\underline{\mathbf{M}}$ is given by :

$$
\underline{\mathbf{M}}=\frac{1}{\rho^{n-1}}\left(\mu \underline{\mathbf{K}}^{-1}+\frac{F}{r}\left|\mathbf{G}^{n-1}\right| \underline{\mathbf{I}}\right) .
$$

Finally, the problem without convection can be written in variational form as follows :

$$
\left\{\begin{array}{l}
\text { Find } x_{1} \in X_{1}^{*} \\
\mathcal{A}_{1}\left(x_{1}, x_{1}^{\prime}\right)=\mathcal{F}_{1}\left(x_{1}^{\prime}\right), \forall x_{1}^{\prime} \in X_{1}^{0}
\end{array}\right.
$$

where $x_{1}=\left(\mathbf{G}_{1}, \mathbf{q}_{1}, p_{1}, T_{1}\right)$ belongs to $X_{1}=\left(H\left(\operatorname{div}, \Omega_{1}\right)\right)^{2} \times\left(L^{2}\left(\Omega_{1}\right)\right)^{2}$ and where $\mathcal{A}_{1}$ is a non-classical mixed operator. $X_{1}^{0}$ and $X_{1}^{*}$ are subsets of $X_{1}$, which take into account the homogeneous, respectively non-homogeneous boundary conditions.

Problem (1.1) as well as the complete problem with convection were shown to be well-posed in [1], where a finite element discretization based on Raviart-Thomas elements for the fluxes and piecewise constant elements for the scalar unknowns was also proposed. We refer to [1] for the numerical analysis and the validation of this scheme.

2. The 1.5D wellbore model. We consider a vertical wellbore, governed by the mass and the energy conservation laws, the compressible Navier-Stokes equations together with the Peng-Robinson state equation.

As for the reservoir, the problem is written in $2 \mathrm{D}$ axisymmetric form and two conservative variables (the specific flux $\mathbf{G}=\rho \mathbf{u}$ and the heat flux $\mathbf{q}=\lambda \nabla T$ ) are 
introduced. The time-discretization yields a nonlinear decoupled system which is solved, at each time step, by a fixed point method with respect to $\rho$. More precisely, for a given $\rho$, we compute the specific flux from the mass equation by means of a Petrov-Galerkin method :

$$
\operatorname{div}(r \mathbf{G})=-r \frac{\rho-\rho^{n-1}}{\Delta t}
$$

then we calculate the velocity and the pressure by solving the following quasi-Stokes system :

$$
\left\{\begin{array}{l}
\operatorname{div}(r \mathbf{u})=\frac{1}{\rho}\left(\operatorname{div}(r \mathbf{G})-\frac{r}{\rho} \mathbf{G} \cdot \nabla \rho\right) \\
r \rho \frac{\mathbf{u}}{\Delta t}+r \mathbf{G} \cdot \nabla \mathbf{u}+r \nabla p-\operatorname{div}(r \underline{\tau})+\tau_{\theta \theta} \mathbf{e}_{r}+r \kappa|\mathbf{G}| \mathbf{u}=r \rho \mathbf{g}+r \rho \frac{\mathbf{u}^{n-1}}{\Delta t}
\end{array}\right.
$$

and we evaluate the heat flux and the temperature from the energy system :

$$
\left\{\begin{array}{l}
r c_{v}\left(\rho \frac{T}{\Delta t}+\mathbf{G} \cdot \nabla T\right)-\operatorname{div}(r \mathbf{q}) \\
\quad=r \rho c_{v} \frac{T^{n-1}}{\Delta t}-\frac{1}{2} r\left(\rho \frac{|\mathbf{u}|^{2}-\left|\mathbf{u}^{n-1}\right|^{2}}{\Delta t}+\mathbf{G} \cdot \nabla\left(|\mathbf{u}|^{2}\right)\right)-\operatorname{div}(r p \mathbf{u})+\operatorname{div}(r \underline{\tau} \mathbf{u})+r \mathbf{g} \cdot \mathbf{G} \\
\mathbf{q}=\lambda \nabla T .
\end{array}\right.
$$

Here above, $\kappa$ is a friction coefficient depending on the diameter of the pipe, $c_{v}$ is the specific heat while $\underline{\tau}$ is the viscous stress tensor of the fluid. Finally, we update the density and we loop until convergence is achieved.

Concerning the boundary conditions, the specific flux, the pressure and the temperature are imposed on the perforations while adiabatic and impermeability conditions are set on the bottom and on the lateral boundary. Note that when considering the sole wellbore problem, one cannot impose the flowrate at the pipe's surface, which is an outflow boundary for the transport equation satisfied by G. This drawback disappears when solving the coupled problem.

In order to take into account the privileged direction of the flow and to reduce the computational cost, a $1.5 \mathrm{D}$ model is derived as a conforming approximation of the $2 \mathrm{D}$ one, by constructing an explicit solution in terms of the radial coordinate. More precisely, $\mathbf{u}, \mathbf{G}$ and $\mathbf{q}$ are taken affine with respect to $r$ while $p, T$ and $\rho$ are taken independent of $r$. Thus, one can now treat pipes of several thousands meters height but of radius $R \simeq 0.15 \mathrm{~m}$ without any numerical instabilities due to this large aspect ratio.

The space discretization is achieved on a rectangular mesh which consists of only one cell in the radial direction. We employ Raviart-Thomas finite elements for the heat and mass fluxes, $Q_{0}$ elements for the pressure and the temperature and $Q_{1}$ continuous elements for the velocity. A detailed proof of the well-posedness of the continuous and discrete problems as well as numerical tests validating the model can be found in [2].

Finally, in view of the coupling, we linearize the momentum and energy equations with respect to $\mathbf{G}$. Then the wellbore problem can be written in weak form as follows :

$$
\left\{\begin{array}{l}
\text { Find } x_{2} \in X_{2}^{*} \\
\mathcal{A}_{2}\left(x_{2}, x_{2}^{\prime}\right)=\mathcal{F}_{2}\left(x_{2}^{\prime}\right), \forall x_{2}^{\prime} \in Y_{2}^{0}
\end{array}\right.
$$

where $x_{2}=\left(\mathbf{G}_{2}, \mathbf{q}_{2}, \mathbf{u}_{2}, p_{2}, T_{2}\right)$, respectively $x_{2}^{\prime}=\left(\chi, \mathbf{q}_{2}^{\prime}, \mathbf{u}_{2}^{\prime}, p_{2}^{\prime}, T_{2}^{\prime}\right)$ belong to : $X_{2}=\left(H\left(\operatorname{div}, \Omega_{2}\right)\right)^{2} \times \mathbf{H}^{1}\left(\Omega_{2}\right) \times\left(L^{2}\left(\Omega_{2}\right)^{2}, \quad Y_{2}=L^{2}\left(\Omega_{2}\right) \times H\left(\operatorname{div}, \Omega_{2}\right) \times \mathbf{H}^{1}\left(\Omega_{2}\right) \times\right.$ $\left(L^{2}\left(\Omega_{2}\right)\right)^{2}$. 
3. Coupling of the previous models. We are now interested in the coupled reservoir-wellbore problem, which is different from those currently considered in the literature. Besides the fact that we deal with a 2.5D - 1.5D coupling, the wellbore also has an additional unknown compared to the reservoir. Moreover, the density is not constant in the two media and the energetic aspect is also taken into account.

In order to bind together problems (1.1) and (2.1), we have to introduce adequate transmission conditions at the perforations. Let $\mathbf{n}$ denote the normal unit vector to $\Sigma$, oriented from the reservoir to the wellbore. Then we impose :

$\mathbf{G}_{1} \cdot \mathbf{n}=R \mathbf{G}_{2} \cdot \mathbf{n}, \quad \rho_{2} \mathbf{u}_{2} \cdot \mathbf{n}=\mathbf{G}_{\mathbf{2}} \cdot \mathbf{n}, \quad \mathbf{q}_{1} \cdot \mathbf{n}=R \mathbf{q}_{2} \cdot \mathbf{n}, \quad-p_{1}=-p_{2}+\underline{\tau}_{2} \mathbf{n} \cdot \mathbf{n}, \quad T_{1}=T_{2}$.

Moreover, due to the viscous context, the tangential velocity of the fluid must also be prescribed. For the sake of simplicity, here we take $\mathbf{u}_{2} \cdot \mathbf{t}=0$ but one could also consider the Beavers-Joseph-Saffman law (see for instance [5] and references therein).

In order to take into account the recorded flowrates at the pipe's surface, a global resolution at each time step is envisaged. Therefore, the transmission conditions are dualized by means of Lagrange multipliers and a global mixed formulation is derived (see also [5]). More precisely, we introduce the spaces :

$$
\begin{array}{lll}
\mathbb{X}=\left\{x=\left(x_{1}, x_{2}\right) \in X_{1} \times X_{2} ; \mathbf{G}_{1} \cdot \mathbf{n}, \mathbf{q}_{1} \cdot \mathbf{n} \in L^{2}(\Sigma)\right\}, & \mathbb{L}=\left(L^{2}(\Sigma)\right)^{2}, \\
\mathbb{Y}=\left\{x^{\prime}=\left(x_{1}^{\prime}, x_{2}^{\prime}\right) \in X_{1} \times Y_{2} ; \mathbf{G}_{1} \cdot \mathbf{n}, \mathbf{q}_{1} \cdot \mathbf{n} \in L^{2}(\Sigma)\right\}, & \mathbb{K}=\left(L^{2}(\Sigma)\right)^{3},
\end{array}
$$

and the bilinear forms on $\mathbb{L} \times \mathbb{Y}$, respectively on $\mathbb{K} \times \mathbb{X}$ :

$$
\begin{aligned}
\mathcal{I}\left(\Lambda, x^{\prime}\right) & =\int_{\Sigma}\left(\mathbf{G}_{1}^{\prime} \cdot \mathbf{n}-R \mathbf{u}_{2}^{\prime} \cdot \mathbf{n}\right) \theta d \sigma-\int_{\Sigma}\left(\mathbf{q}_{1}^{\prime} \cdot \mathbf{n}-R \mathbf{q}_{2}^{\prime} \cdot \mathbf{n}\right) \mu d \sigma \\
\mathcal{J}\left(\Lambda^{\prime}, x\right) & =\int_{\Sigma}\left(\mathbf{G}_{1} \cdot \mathbf{n}-R \rho_{2} \mathbf{u}_{2} \cdot \mathbf{n}\right) \theta^{\prime} d \sigma \\
& +\int_{\Sigma}\left(\mathbf{G}_{1} \cdot \mathbf{n}-R \mathbf{G}_{2} \cdot \mathbf{n}\right) \zeta^{\prime} d \sigma-\int_{\Sigma}\left(\mathbf{q}_{1} \cdot \mathbf{n}-R \mathbf{q}_{2} \cdot \mathbf{n}\right) \mu^{\prime} d \sigma .
\end{aligned}
$$

We can now consider the following weak formulation of the coupled problem :

$$
\begin{cases}\text { Find } x \in \mathbb{X}^{*}, \Lambda \in \mathbb{L} & \\ \mathcal{A}\left(x, x^{\prime}+\mathcal{I}\left(\Lambda, x^{\prime}\right)\right. & =\mathcal{F}\left(x^{\prime}\right), \forall x^{\prime} \in \mathbb{Y}^{0} \\ \mathcal{J}\left(\Lambda^{\prime}, x\right) & =0, \quad \forall \Lambda^{\prime} \in \mathbb{K}\end{cases}
$$

where $\mathcal{A}\left(x, x^{\prime}\right)=\mathcal{A}_{1}\left(x_{1}, x_{1}^{\prime}\right)+\mathcal{A}_{2}\left(x_{2}, x_{2}^{\prime}\right), \mathcal{F}\left(x^{\prime}\right)=\mathcal{F}_{1}\left(x_{1}^{\prime}\right)+\mathcal{F}_{2}\left(x_{2}^{\prime}\right)$. The multiplier $\Lambda=(\theta, \mu)$ can be interpreted as $\left(p_{1}, T_{1}\right)$ or $\left(p_{2}-\tau_{r r}, T_{2}\right)$ and $\mathcal{J}$ dualizes the continuity of the fluxes. .

The global operator $\mathcal{A}$ is of mixed type, $\mathcal{A}=\left[\begin{array}{cc}A & B \\ -B^{T} & C\end{array}\right]$ with :

$$
\begin{aligned}
A\left(x, x^{\prime}\right)= & \int_{\Omega_{1}} \frac{1}{r} M \mathbf{G}_{1} \cdot \mathbf{G}_{1}^{\prime} d x+\int_{\Omega_{1}} \frac{1}{r \lambda_{1}} \mathbf{q}_{1} \cdot \mathbf{q}_{1}^{\prime} d x+\int_{\Omega_{2}} \chi \operatorname{div}\left(r \mathbf{G}_{2}\right) d x+\int_{\Omega_{2}} \frac{r}{\lambda_{1}} \mathbf{q}_{2} \cdot \mathbf{q}_{2}^{\prime} d x \\
& +\int_{\Omega_{2}} r\left(\frac{\rho_{2}}{\Delta t}+\kappa\left|\mathbf{G}_{2}\right|\right) \mathbf{u}_{2} \cdot \mathbf{u}_{2}^{\prime} d x+\int_{\Omega_{2}} r\left(\mathbf{G}_{2} \cdot \nabla\right) \mathbf{u}_{2} \cdot \mathbf{u}_{2}^{\prime} d x, \\
B\left(x, x^{\prime}\right)= & -\int_{\Omega_{1}} p_{1} \operatorname{div} \mathbf{G}_{1}^{\prime} d x+\int_{\Omega_{1}} T_{1} \operatorname{div} \mathbf{q}_{1}^{\prime} d x-\int_{\Omega_{2}} p_{2} \operatorname{div}\left(r \mathbf{u}_{2}^{\prime}\right) d x+\int_{\Omega_{2}} T_{2} \operatorname{div}\left(r \mathbf{q}_{2}^{\prime}\right) d x, \\
C\left(x, x^{\prime}\right)= & \int_{\Omega_{1}} \frac{r}{\Delta t}\left(a p_{1}-b T_{1}\right) p_{1}^{\prime} d x+\int_{\Omega_{1}} \frac{r}{\Delta t}\left(d T_{1}-f p_{1}\right) T_{1}^{\prime} d x+\int_{\Omega_{2}} r \frac{c_{v} \rho_{2}}{\Delta t} T_{2} T_{2}^{\prime} d x .
\end{aligned}
$$

Concerning the approximation of (3.1), we assume that the two meshes are matching at the interface and we employ the previously introduced finite element spaces for $x$, while $\Lambda$ is taken piecewise constant. The convective terms are treated by appropriated upwind schemes. 
The well-posedness of both the continuous and discrete problems is established in [3]. Note that the mathematical analysis of (3.1) is nonstandard, since $A$ and $C$ are non symmetric and since the spaces of the solution and of the test-functions are different. The operator $\mathcal{A}$ was shown to satisfy a first inf-sup condition, thus ensuring the uniqueness thanks to Babuška's theorem. However, the proof of the second inf-sup condition is, at our knowledge, an interesting open problem. We have then managed to establish the existence of a solution in the continuous case by means of a Galerkin method, using the existence in the discrete case.

4. Numerical tests. In order to validate the code from both a numerical and a physical point of view, we consider here a reservoir consisting of two producing layers characterized by homogeneous physical and thermodynamic properties $\left(k_{h}=\right.$ $130 \mathrm{mD}, k_{v}=1 \mathrm{mD}, \phi=0.15$ and $S_{w}=0.2$ ) and separated by a quasi-wall (with $k_{h}=0.01 m D, k_{v}=0.01 m D, \phi=0.1$ and $\left.S_{w}=0.95\right)$, where $k_{h}, k_{v}$ represent the horizontal, respectively vertical permeabilities while $S_{w}$ is the water's saturation. We simulate the production of a light oil during two days by imposing constant pressure and temperature $\left(p_{\gamma}=261 \mathrm{bar}, T_{\gamma}=402 \mathrm{~K}\right)$ on the external boundary of the reservoir and a constant flowrate at the pipe's surface $\left(Q=1500 \mathrm{~m}^{3} / j\right)$. All previous data are realistic.

As regards the behavior of the solution with respect to mesh refinement, every triangle in the reservoir is successively divided into four congruent ones and every rectangle in the well into 2 congruent ones. For every intermediate mesh, we have evaluated the $L^{2}$-error between the current solution and the reference one, computed on the finest mesh. We have thus obtained the following convergence rates for the pressure, repectively the temperature : $O\left(h^{1.39}\right)$ in the reservoir and $O\left(h^{1.61}\right)$ in the wellbore, respectively $O\left(h^{1.1}\right)$ and $O\left(h^{1.56}\right)$.

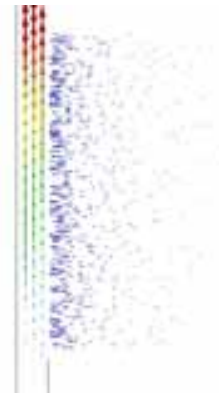

(a) Specific flux

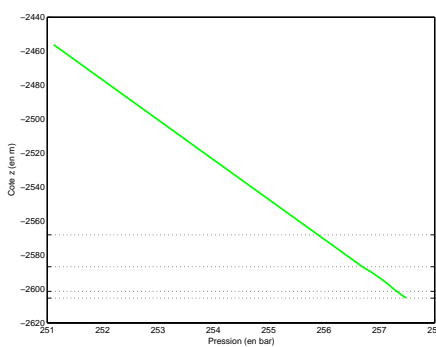

(b) Pressure in the well

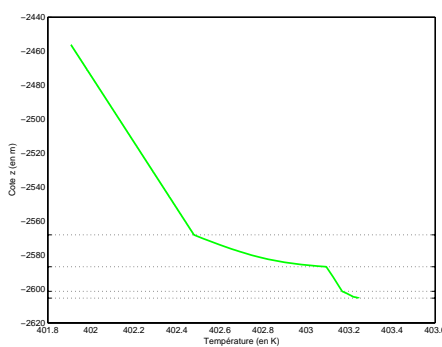

(c) Temperature in the well

FIG. 4.1. Specific flux, pressure and temperature at the end of the production.

In figure 4.1(a) we have represented the specific flux in the upper layer of the reservoir and in the well. One may note that we recover the fact that the velocity in the wellbore is much more important than the one in the reservoir, due to the flux conservation. In order to better visualise the flow near the perforations, different scalings were used in the two domains (of ratio equal to 10). Figures 4.1(b) and 4.1(c) show the pressure and the temperature maps in the wellbore at the end of the production. One retrieves a linear pressure (primarily influenced by the gravity) and a linear temperature above the perforations. Finally, we present in Figure 4.2 the evolution of the pressure and the temperature in the whole domain during two days, since afterwards the flow almost reaches the steady state. We focus on the neighbourhood of the per- 
forations : only $6 \mathrm{~m}$ of the reservoir are visualised. The numerical results correspond to the physical behavior expected by the petroleum engineers, and equally to the results given by each separate code. In particular, one may note that the transmission conditions at the interface are satisfied. A decrease of the pressure in the producing layers and the wellbore is noticed while an increase of the temperature due to the Joule-Thomson effect can be observed.

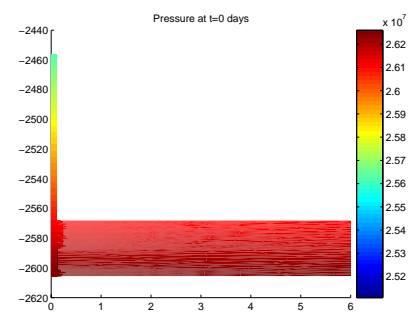

(a) Pressure at $\mathrm{t}=0$ day

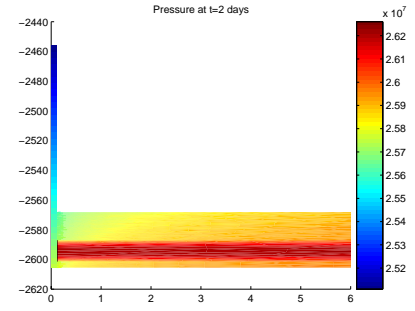

(c) Pressure at $\mathrm{t}=2$ days

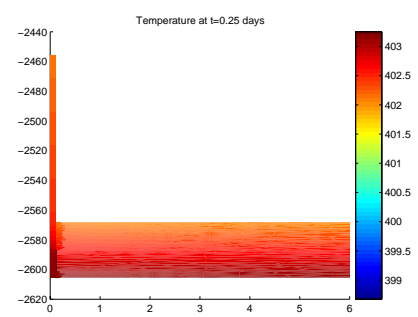

(e) Temperature at $\mathrm{t}=0.25$ day

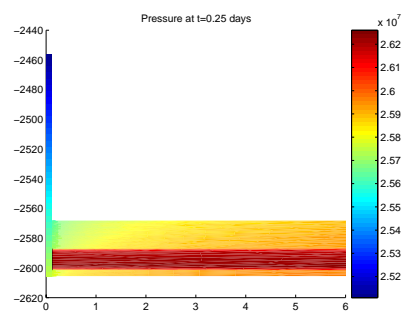

(b) Pressure at $\mathrm{t}=0.25$ day

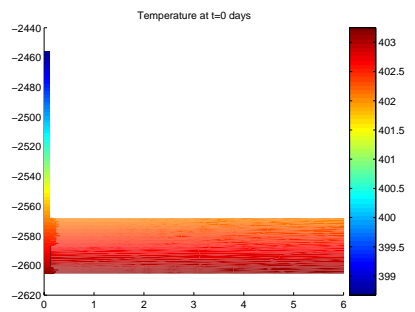

(d) Temperature at $\mathrm{t}=0$ day

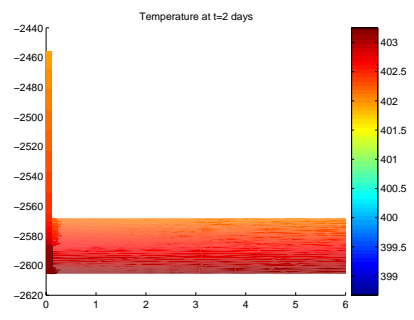

(f) Temperature at $\mathrm{t}=2$ days

FIG. 4.2. Behaviour of the pressure and temperature during two days production.

\section{RÉFÉRENCES}

[1] Amara M, Capatina D, Denel B, Terpolilli P. Mixed finite element approximation for a coupled petroleum reservoir model. M2AN $2005 ; 39(2): 349-376$.

[2] Amara M, Capatina D, Denel B. A 1.5D Petroleum Wellbore Model with Heat Transfer. http ://lma.univ-pau.fr/publis/publis_pre.php [2007].

[3] Amara M, Capatina D, Lizaik L. Coupling of a Darcy-Forchheimer model and compressible Navier-Stokes equations with heat transfer. Submitted to SIAM J.Sci.Comp. 2007.

[4] Maubeuge F, Didek M, Arquis E, Bertrand O, Caltagirone J.P. A model for interpreting thermometrics. SPE $1994 ; \mathbf{2 8 5 8 8}$.

[5] Layton W, Schiewek F, Yotov I. Coupling fluid flow with porous media flow. SIAM J. Numer. Anal. $2003 ;$ 40(6) :2195-2218. 
[6] Forchheimer P. Wasserbewegung durch Boden. Z. Ver. Deutsh. Ing. 1901; 45 :1782-1788.

[7] Peng D.Y, Robinson D.B. A new two-constant equation of state; Ind. Eng. Chem. Fundam. $1976 ; 15: 59-64$.

[8] Pinzon I.D, Davies J.E, Mammadkhan F, Brown G.A. Monitoring Production From GravelPacked Sand-Screen Completions on BP's Azeri Field Wells Using Permanently Installed Distributed Temperature Sensors. SPE 2007; 110064. 